\title{
Design for Reliability Based Methodology for Automotive Gearbox Load Capacity Identification
}

\author{
Milosav Ognjanovic ${ }^{1}$ - Miroslav Milutinovic ${ }^{2,}$ \\ ${ }^{1}$ University of Belgrade, Faculty of Mechanical Engineering, Serbia \\ ${ }^{2}$ University of East Sarajevo, Faculty of Mechanical Engineering, Bosnia and Herzegovina
}

Robust and Axiomatic design, a property-based approach in design, is applied and integrated into a new methodology for developing Functional Requirements (FR) or Design Parameters (DP). The reliability of the design structure and the elementary reliability of the design components are used as a functional requirement of the automotive gearbox, in relation to the service life and operation conditions, and also as a design constraint in analytical relationships. For this purpose, elementary reliability and allowable stress are defined in a specific way. The automotive gearbox, operating under varying and random operation conditions, is used as a case study. The same design structure has to operate under different operation conditions. In these circumstances, the carrying capacity as a functional requirement is related to the operation conditions and operation regime. The model presented in this paper, as well as a computer program, enable identification of this carrying capacity. This paper discusses an interdisciplinary and multi-methodological integrated approach to the presented task. Experimental data regarding the failure probability of gearbox components and the probability of operation conditions processing, the decomposition of gearbox structure, and the elementary reliability treatment as a component of design property are only some of the methods applied.

Keywords: robust design, axiomatic design, gearbox, reliability

\section{INTRODUCTION}

The functional requirements of design structures, for example, the load capacity, reliability, service life, etc. are strongly related to operation conditions that are, as a rule, random and very often uncertain. The basic idea is to apply a set of methods in Engineering Design in order to develop a methodology for load capacity identification with regard to operation conditions. An automotive gearbox has been taken for the case study, because it represents a design structure with very variable operation conditions. The reliability of structures such as gearboxes is one of the main indicators of quality and an important functional requirement. In order to fulfil this objective, various methods and approaches are taken into consideration, such as Robust and Axiomatic Design, Property-based design, Design for X, and various studies in the fields of automotive gearboxes, gears and gear failures, the reliability of gear transmission components, etc.

Today, Axiomatic design provides a basis for analytic presentation of the relationship between functional requirements and design parameters. The Axiomatic principles developed by Suh, continue to perfectly for various applications [1], but this methodology is also suitable for combining with the Robust Design approach. On the other hand, the Property-based design approach together with the system structure and functional requirements or quality indicators deduction provides possibilities for arranging specific procedures and approaches for the design parameters or the carrying capacity identification for design structures such as automotive gearboxes. Robust Design is a verified methodology, which provides good results on the first attempt. It also provides good design results (the relationship between functional requirements and design parameters) under variable operation conditions, and is applied for solving problems in many kinds of specific design cases. For horizontal axis wind turbines, the Robust design is carried out using the Taguchi method [2] and multi-objective optimization of the design parameters. The robustness of uncertain design variables can be provided by optimization using evolutionary algorithms [3]. The optimization is performed by analysing whether the solutions are truly robust and how robust optimal solutions differ from the performance maximizing solutions. Further perfection of robustness is provided for quality variables that vary over time [4]. Using the expectation of maximal quality loss over a time period, this work suggests the possibility of quantifying the robustness for timedependent quality characteristics. The reliability of technical systems such as automotive gearboxes is a typical time-dependent quality indicator that has to be treated in a similar way. In addition, the desired elementary reliability at the end of the service life of a design component can be applied as a design constraint for the design parameters definition [5] and [6].

The reliability of technical systems has been studied for a long time, basically for the sake of system maintenance, and has been oriented in two directions. The first direction is a continuation of the research for 
maintenance necessities, such as reliability prediction [7], short-term reliability analysis of power generating units [8] in order to identify the actual reliability level of the power-generating unit, or the application of Natvig measures in the reliability identification of repairable systems [9]. The other direction is oriented towards the necessity for product development and design. This can be used for direct applications such as design optimization [10] in conditions of nonGaussian distribution, identification of reliability decrease in the course of service life [11] or investment analysis for increasing reliability of the technical system [12]. The decomposition of a technical system into components and the decomposition of their functional requirements and indicators of quality is an important step in both the reliability analysis and the design parameters definition. The decomposition of the system and relationships into a transformation matrix enables application of the axiomatic approach or determines the reliability of a certain component analysis. Very complex structures such as a vehicle [13] can be decomposed down to the level of design modules (engine, transmission, break system, etc.) and then into the level of their components (gears, bearings, coupling, etc.). The decoupling of the transformation matrixes is the main task of this process and the results are applicable in solving various design problems, such as stochastic problems in reliability [14], design parameters, functional requirements, and the quality indicators definition. Decomposition is also used in gear teeth wear analysis [15] and in the design components' failure propagation [16].

The Design for X (DFX) [17] is oriented towards a certain aspect of product analysis or synthesis, which is the opposite of holistic or integrated approaches to product development and design. The property-based approach in design also takes into consideration a chosen property and provides the possibility of solving a certain problem in design [18]. The "Design for $\mathrm{X}^{\text {" was }}$ the proposed implementation platform used in rapidly configuring the optimal design solution based on the company's requirements in the case of refrigerators [19]. Further analysis continues with the property-based approach, where the elementary reliability of the structure component is the property used as a design constraint in the load capacity and design parameters identification. This is a continuation of articles [20] and [21]. The established relationships can then be used for direct or reverse engineering such as [22]. In order to connect automotive gearboxes as in case study [23], the results for the torque measurements of these structures [24] and [25], as well as torque based vehicle speed control [26], are used.

This paper offers a new and specific approach based on the integration of axiomatic and robust methodology directed towards load capacity identification of the existing design structure or the design parameters definition in the design process of the new mechanical structure. Reliability plays multiple roles, as a: functional requirement, design property, design constraint, indicator of quality, and indicator of design behaviour. Compared to other design optimisation approaches, the advantage of this particular one is the possibility of controlling the probabilistic variation in operation conditions and the fulfilment of design robustness, i.e. reaching design parameters or functional requirement (load capacity) insensitivity to the variation in operation conditions.

\section{PROBLEM DEFINITION}

Basically, the main task of the design process is to create a relationahip between the product function, product structure, and product behaviour. The product function explains the effects of the system and creates a correlation between the input conditions and output effects. Product behaviour represents the interaction of the function with the environment and how the product fulfils its function. This is a result of the properties and characteristics of the assemblies and parts in relation to the environment and system use. In order to fulfil the functional requirements and to obtain the desired system behaviour, it is necessary to create a satisfactory design structure combining components (mechanical, electrical, information, etc.) in corresponding assemblies and system. One of the models for product structure creation is the $\mathrm{V}$-model established by VDI-2206 as the "Design methodology of mechatronic systems" which contains a synthesis of the system in order to obtain a design structure based on functional requirements, and an analysis aimed at integrating the system behaviour. This means a synchronized process of design structure processing and a functional requirements' transformation into design structure behaviour. For this approach, known as Property-based design in [18], a monitoring system is established to provide the desired system behaviour starting from the defined functional requirements (Fig. 1).

The reliability of technical systems is an important indicator of the system's quality. In the clarification of the tasks design stage, reliability is one of the main functional requirements, and, in the operation process of a technical system, reliability is one of 


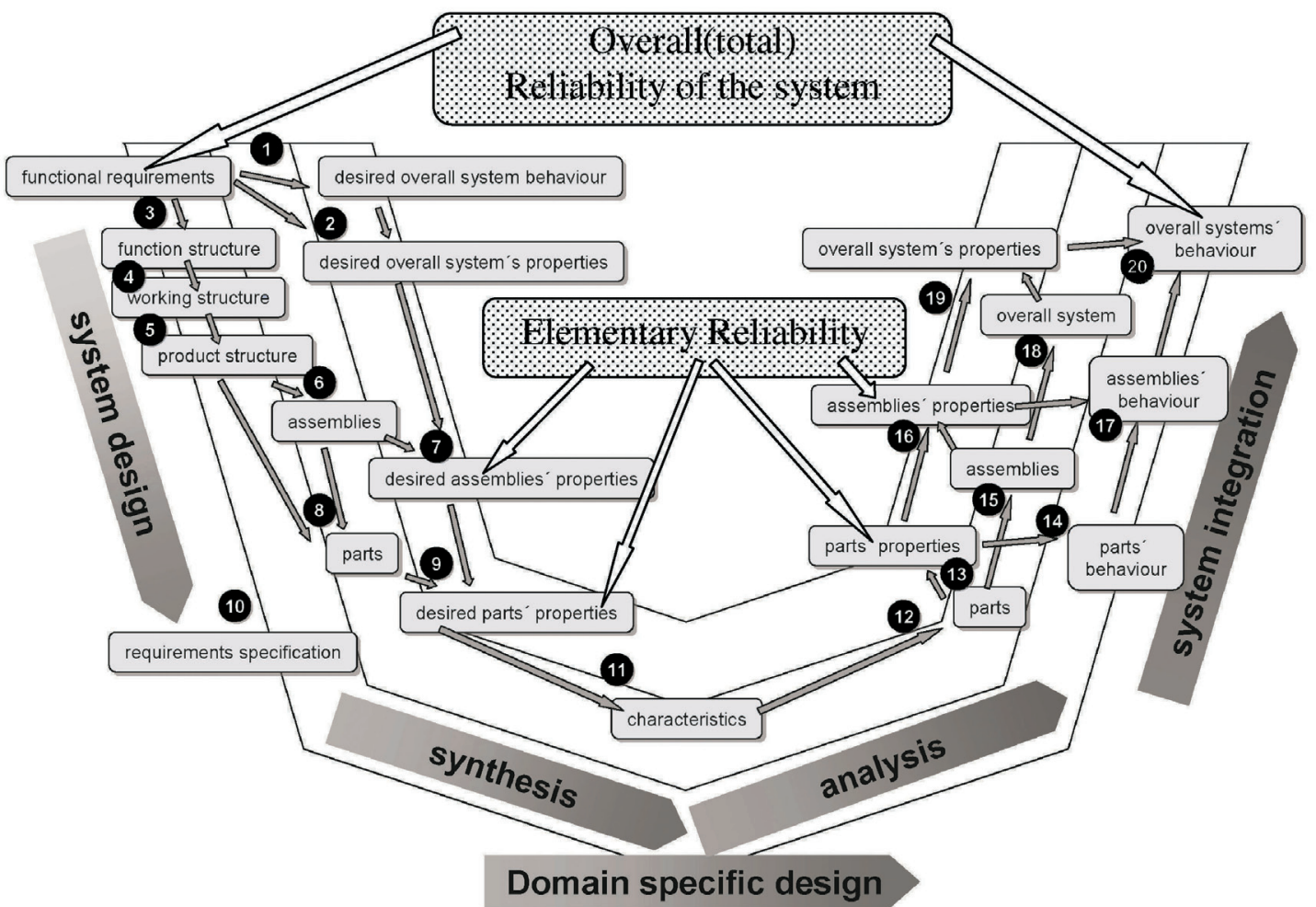

Fig. 1. V-Model of design process addapted for the property based product development with reliability as functional requirement, design property and as an indicator of system behaviour

the main indicators of quality and system behaviour. By decomposing the desired reliability of the system (functional requirement) to the design component level, the elementary reliability becomes the design property of the component (Fig. 1). According to [18], design properties of the design components are the result of the parts properties (intensive and extensive) and parts characteristics. These characteristics are the physical and chemical description of the material, geometrical (shape, dimensions,.etc.) and structural (joints and parts) interactions.

Elementary reliability as a design property is a specific term in this paper, characterized by the following: (a) In the reliability system hierarchy it is connected to the technical system's basic component. (b) As there is more than one possible failure in one component, the elementary reliability is connected to the top probable failure only. (c) This is a complex probability that contains the probability of operation conditions and the failure probability for certain operation conditions (see the case of gears in Eq. (1)).

Reliability is one of the functional requirements of the system. An automotive gearbox is chosen as a case study for the presented approach, because it is complex in the functional, structural, and behavioural regard. A set of functional requirements contains the load capacity, number of speed levels, reliability level, volume and mass value, vibration and noise level, handling suitability, etc. Load capacity is the main indicator of gearbox design and as such is the main aim of this paper and the developed approach. For the purpose of applying the axiomatic and robust design approach in further processing, the abovementioned group of functional requirements is divided into two groups. In order to simplify the axiomatic relationship, the load capacity, volume, and number of transmission stages are used as functional requirements. The rest of the functional requirements, such as reliability, vibration and noise level, are used for the design constraints definition in order to fulfil the robustness of the design process. The section on carrying (load) capacity identification will discuss this in more detail. For a given technical system (for example, the automotive gearbox), functional requirements can also be used as design constraints (Fig. 2) for the purpose of applying both the axiomatic and robust design. Members of matrix A are directly related to constraints related to operation conditions and selected functional requirements. In this case, the elementary reliability of gearbox components is the design constraint 
that provides robustness to the design process. The vibration and noise of a gearbox can be used as a design constraint in order to harmonize the interaction of design parameters, but this article does not address this aspect of the design.

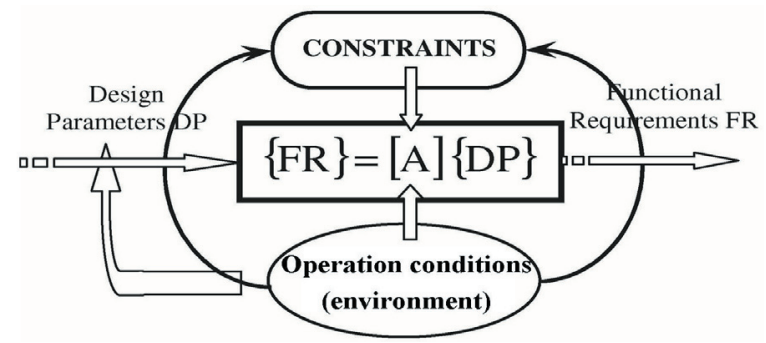

Fig. 2. The relationship of functional requirements $F R$, design parameters $D P$, operation conditions, and constraints in the axiomatic and robust design of automotive gearboxes

\section{STRUCTURING OF THE SYSTEM}

The product model, i.e. the technical system model, displays chosen characteristics or properties in a form suitable for the desired analysis. The model also represents the structure and relationship of these characteristics and properties. The planned automotive gearbox analysis requires a few models, such as a functional one, then a model of the design structure, a model of the parts and assembly geometry, models of reliability of the system, and of elementary reliability, etc. A model of gearbox function is presented in Fig. 3. This is a gearbox with six speeds and an additional one for reverse motion. Input speed is transformed into slow speed and then for every output speed transforms back to a corresponding speed level. The last, sixth speed, is equal to input speed without transformation. Output speed for reverse motion is additionally reduced and the direction is reversed. The transformation of input power carries out the function marked by 0 and then for the I to $\mathrm{V}$ speed levels an additional transformation is carried out for the functions marked by 1 to 5 and also for the reverse motion by the function labelled $\mathrm{R}$. The maximal speed level VI is the input speed without transformation. The control and choice of speed level is provided by the steering system with couplings $\mathrm{c} 1$ to $\mathrm{c} 3$ and $\mathrm{cR}$, in interaction with the vehicle driver.

The function structure transformed into organ structure (conceptual design) can be presented as shown in Fig.4. This structure consists of three shafts: the input, output and middle shaft. Gear pairs $j=0,1$, $2, \ldots, \mathrm{R}$ are located on these shafts together with the couplings. The output and input shafts are coaxial and all gear pairs have the same centre distances.

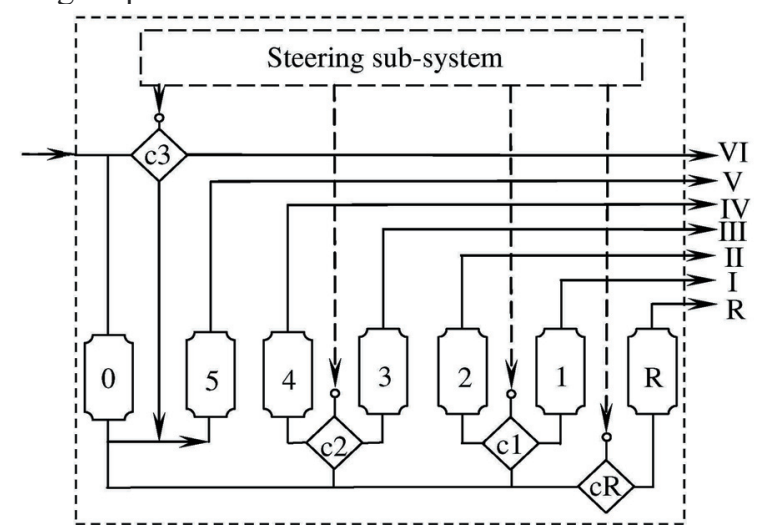

Fig. 3. The automotive gearbox function structure

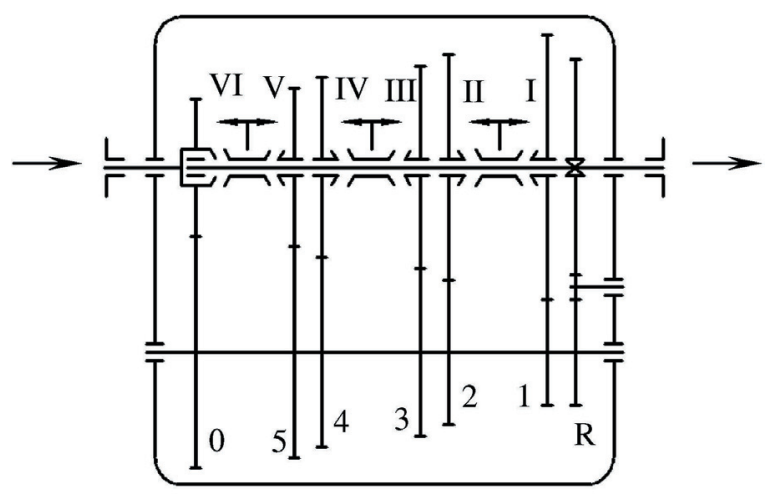

Fig. 4. Organ structure (conceptual design) of the automotive gearbox

The structure of design components and assemblies is presented in Fig. 5. This is a 3D geometric model of a complete gearbox and its component representatives, the gear pair, coupling, shaft, bearing, eagling, etc. This structure allows one to create a reliability structure model. Compared to other reliability models, the model for axiomatic and robust design has to be created according to the following procedure and limitations.

The technical system, i.e. the automotive gearbox, has to have the ability to be decomposed into components that can be completely replaced in case of damage. These are (Fig. 5): seven gear pairs, three couplings, five bearings, and two seals. In Fig. 6 , a separate elementary reliability is recognized for each of these as a design property of the component (sub-assembly). The steering ( $s t$ ) sub-system is a specific assembly and $R_{s t}$ is avoided in this analysis of reliability. Total reliability $R$ is the product of component reliabilities, i.e. elementary reliabilities $R_{g j}, R_{b j}, R_{c j}, R_{s e j}$. 


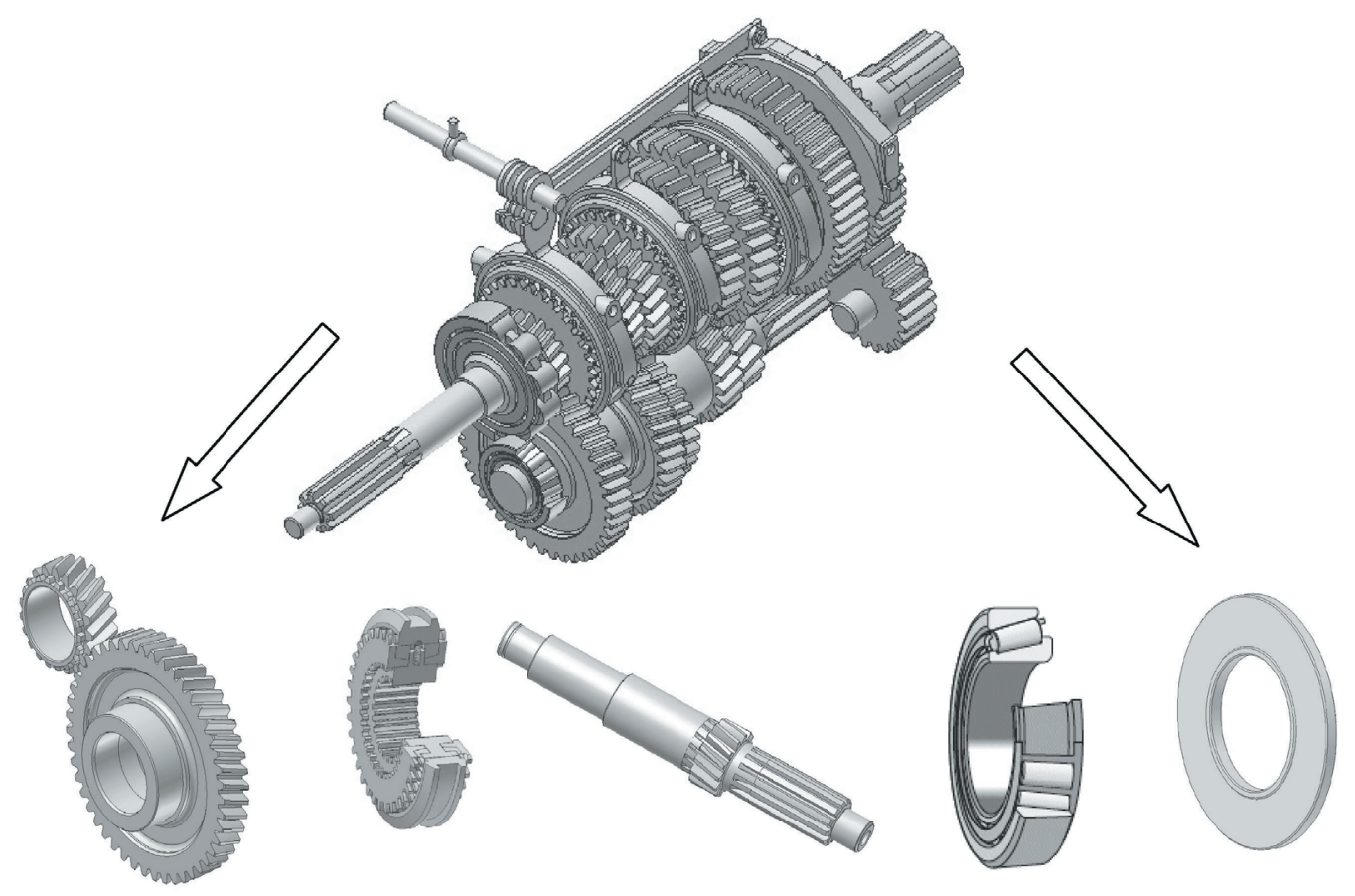

Fig. 5. 3D model of automotive gearbox and its components used for load measurement and for case study

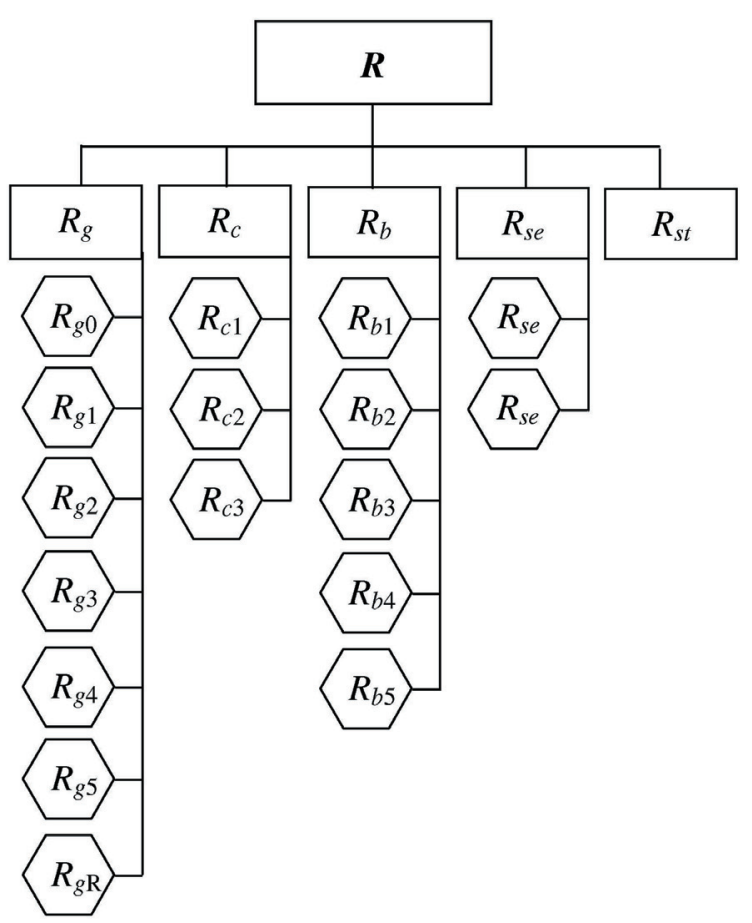

Fig. 6. Model of automotive gearbox reliability

The reliability of design components can also be complex. One component can show two or more types of damage. The teeth of gear pairs can be damaged by wear or by teeth fracture, couplings by the damage of movable parts, and by the damage of the parts for the fixing position, etc. When there is a possibility of more than one failure in a component, the reliability of the component is equal to the lowest reliability of all elementary reliabilities for every failure separately. For example, the elementary reliability of the gear pair is equal to the elementary reliability with regard to teeth wear; the reliability of coupling is equal to the reliability with regard to the damage to the fixed position parts, etc.

In this way, the total reliability of the system (gearbox) is decomposed to the level of probable damage to the component (sub-assembly) and presented in Fig. 6. This is a serial reliability structure composed of the above-mentioned gearbox component reliabilities.

\section{A MODEL FOR THE GEAR PAIR ELEMENTARY RELIABILITY CALCULATION}

Elementary unreliability is a complex probability that includes the probability of certain operation conditions (load, stress, etc.) as well as the failure probability of these operation conditions. Elementary unreliability is, in fact, the product of these two probabilities. For example, gear teeth damage can occur if the gear pair is exposed to the load (stress), 
which can be identified by the probability of stress $\sigma_{H i}$ as $p_{i}\left(\sigma_{H i}\right)=n_{\Sigma i} / n_{\Sigma} \quad\left(n_{\Sigma i}\right.$ is stress cycles number of stress $\sigma_{H i}$ and $n_{\Sigma}$, total stress cycles number in the course of service life). A low level of stress does not produce damage, i.e. it is necessary to consider failure probability in relation to the stress level $P_{F i}\left(\sigma_{H i}\right)$. If the gear pair in the course of service life is exposed to stress levels $i=1,2, \ldots, k$, the gear pair elementary unreliability for gear pair $\mathrm{j}$ is integral, that is, a sum of complex probabilities throughout the entire service life, i.e.:

$$
F_{p j}=\sum_{i}^{k} p_{i}\left(\sigma_{H i}\right) P_{F i}\left(\sigma_{H i}\right) .
$$

Elementary reliability against this certain failure is $R_{g j}=1-F_{p j}$. Failure is possible if two conditions are fulfilled: first, that the stress exists and second, that this stress is able to produce damage. If neither of these conditions exist, failure unreliability is close to zero. Therefore, it is necessary to identify the probabilities of both operation conditions, i.e. the operation regime and the failure probability.

\subsection{Gearbox Operation Regime}

A standard procedure for identifying the operation regime does not exist. A combination of measurements under usage conditions and statistical estimations make it possible to determine the operation regime. VDI norms define load spectrum parameters only. In order to identify the operation regime of a chosen type of gearbox, extensive experimental research has been carried out. The first stage of research was processed by applying the method of interview. About 30 drivers with extensive experience driving trucks answered various questions in a specially prepared questionnaire. Fig. 7 presents a diagram that shows the participation of every gearbox speed in the course of vehicle use under various conditions. In mountainous or hilly areas, lower level gearbox speeds predominate in the course of service life, while in flatter areas the middle levels of speed are more common. Under highway conditions, high speeds dominate. Since the vehicles (trucks and buses) do not operate under only one of these conditions, a combination is common. One of the possible combinations is presented in Fig. 7. This combination includes equal participation of mountain, flat ground, and highway conditions, for the purpose of the case study in the next analysis. The robustness of the presented approach is in the various combinations, which affects the value of the identified gearbox load capacity. For instance, the conditions closer to mountain conditions will reduce the identified load capacity and conditions closer to highways will increase the load capacity. These operation conditions are the customer's requirement presented in the form of combination of various exploitation conditions (load spectrum). This is the base for load capacity identification for these (desired) operation conditions.

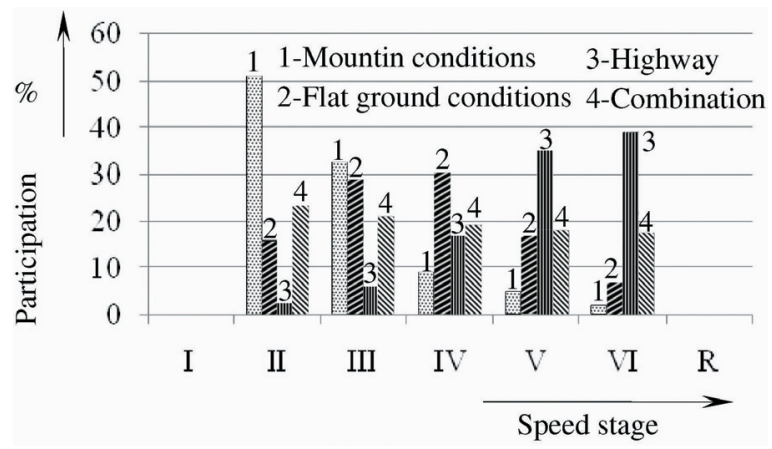

Fig. 7. Participation of gearbox speeds in truck drives under various operation conditions

The second stage of the gearbox operation regime identification is the measurement of output torque under the chosen operation conditions for every gearbox speed. The measurement was carried out using strain gauge transducers at the output shaft of the gearbox. A transducer was connected to the software for data processing using a telemetric transmitter. Table 1 lists the maximum values of the measured torques during the operation.

Table 1. Maximal output torques measured under operation conditions

\begin{tabular}{cc}
\hline Gearbox speeds & Maximal output torque $T_{\text {outp-max }}[\mathrm{Nm}]$ \\
\hline I & 4824 \\
\hline II & 4630 \\
\hline III & 1987 \\
\hline IV & 820 \\
\hline V & 710 \\
\hline VI & 550 \\
\hline R & 4900 \\
\hline
\end{tabular}

By combining statistical samples identified by measurement, interviews and assessment, the arrangement of the total statistical load spectrum of every gearbox stage (speed level) is obtained. The load spectrum shows the participation of gear pair torque in a one million $\left(10^{6}\right)$ gear teeth mesh (Fig. 8). Except for stage VI, gear pair 0 (Figs. 3 and 4) and a corresponding gear pair 1 for stage I, gear pair 2 in stage II, etc. participate in all other stages. Using load spectrums for each transmission stage I, II, III, etc. 
by a corresponding transformation, the load spectrum of each gear pair is arranged and presented in Fig. 8. The ratio $x_{i}=T_{i} / T_{1}$ is equal to 0 to 1 , where $T_{1}$ is the maximal torque of the pinion of the gear pair, $T_{10}$ for gear pair $0, T_{11}$ for gear pair $1, T_{12}$ for gear pair 2 , etc. Torque $T_{i}$ is a variable torque. Maximal torque $T_{1}$ for the pinion of each gear pair is obtained by recalculation (deduction) of the gearbox maximal output torque presented in Table 1.

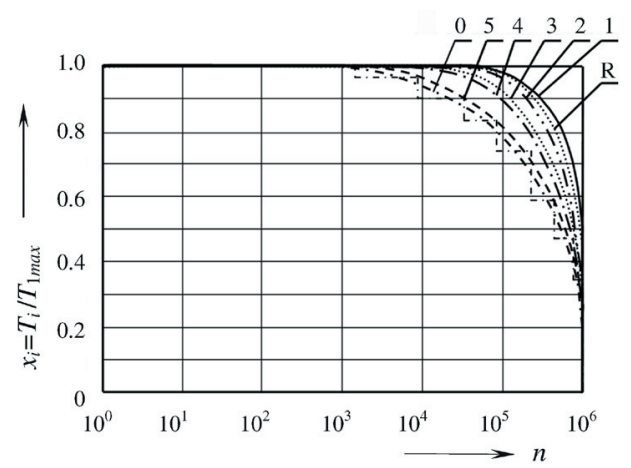

Fig. 8. Load spectrums for the pinions of each gear pair designed based on the measured operation load of the chosen gearbox for gear pairs $j=0,1,2, \ldots, R$.

\subsection{Failure Probability of Gears}

Fig. 9 shows the range of failure probability $\left(P_{F}\right)$ dissipation for gear teeth flanks wear. This range of dissipation is obtained by laboratory testing and defined by ISO 6336 and DIN 3990 for gears made of alloyed steel (15CrNi6) with carbonised teeth flanks. For each stress cycle number $n_{\Sigma i}$, the Weibull function of failure probability is:

$$
P_{F}\left(\sigma_{H}\right)=1-e^{-\left(\frac{\sigma_{H}}{\eta}\right)^{\beta}} .
$$

Parameters of Weibull function $\eta$ and $\beta$ define the bound values from the Fig. 9 diagram for the corresponding stress cycle number $n_{\Sigma i}$ i.e. $\sigma_{H 0.1}$ and $\sigma_{H 0.9}$. These two values replaced in Eq. (2) together with the corresponding failure probability $P_{F}\left(\sigma_{H}\right)=$ 0.1 and $P_{F}\left(\sigma_{H}\right)=0.9$ give equations for calculating variables $\beta$ and $\eta$ :

$\beta=\frac{\log (-\ln 0.1 /-\ln 0.9)}{\log \left(\sigma_{H 0.9} / \sigma_{H 0.1}\right)}, \quad \eta=\frac{\sigma_{H 0.1}}{\sqrt[\beta]{-\ln (1-0.1)}}$. (3)

\section{LOAD CAPACITY IDENTIFICATION}

According to Fig. 2, the robust and axiomatic design of automotive gearboxes is provided by minimizing the necessary information and eliminating the design parameters sensitivity related to operation conditions. Minimum information is obtained by functional requirements and design parameters number minimization. Design parameters connected to the gearbox geometry are parametrically connected with the major parameters such as gear diameters, gear width, gear centre distance, etc. In this way, the number of design parameters responsible for functional requirements is significantly reduced. Other non-geometric DPs are found in transformation matrix A. The number of functional requirements (FR) is also reduced and contains only the major ones, such as gearbox load capacity, speed levels and service life. Operation conditions and level of reliability, together with material characteristics and design properties of the gearbox, are also included in the calculation of the matrix A members.

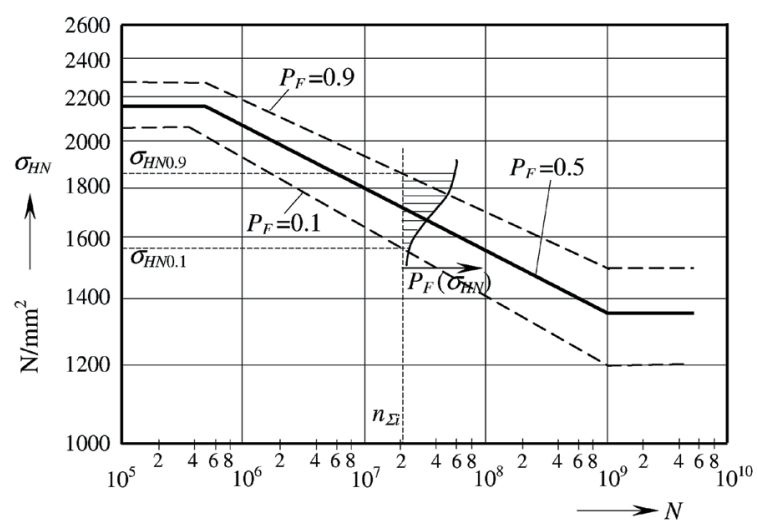

Fig. 9. The range of wear probability distribution of carbonised gear teeth flanks

\subsection{Elementary Reliability of the Gear Pair}

The value that brings into correlation the operation conditions, material characteristics, and design properties randomness is the design allowable stress. The allowable stress also provides robustness to the design process because this value includes possible variations in operation conditions and randomness of characteristics and properties. In order to present its effect, Fig. 10 gives the relationship between the operation load (stress) spectrum and failure probability of gear teeth flanks. Possible variations of operation conditions and failure probability are included in the elementary reliability calculation 
according to Eq. (1). It is possible to carry out a reverse calculation of the design allowable stress level for the desired elementary reliability by using various approaches. One of them is an iterative calculation of reliability with varying operation stresses $\sigma_{H i}$ (keeping the same ratio between them in the spectrum). When the calculated reliability is close to the desired one, the maximum stress in the spectrum represents the design allowable stress $\sigma_{H d e s}=\sigma_{H 1}$.

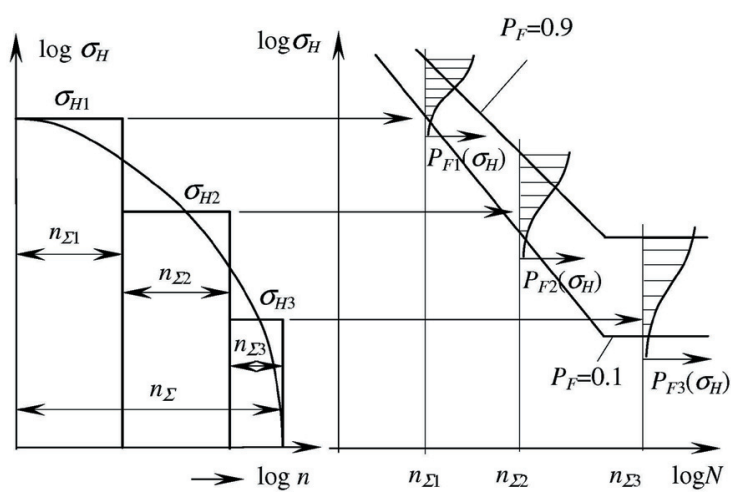

Fig. 10. Operation stress and failure probability range relationship in the elementary reliability calculation of a given gear pair and the design allowable stress $\sigma_{\text {Hdes }}$ definition

Fig. 10 shows that the failure probability and gear pair reliability are strongly correlated with the stress cycle number $n_{\Sigma i}$ in the course of gearbox service life. For the purpose of $\sigma_{\text {Hdes }}$ identification for a certain gear pair, the transformation of load spectrum in Fig. 8 for the corresponding gear pair is necessary. The transformation contains a few steps. The first one is the calculation of Hertzian stress $\sigma_{H}$ according to Eq. (4), i.e. the transformation of the maximum pinion torque $T_{1}$ into maximal stress $\sigma_{H 1}$. The next step is the transformation of the continual logarithmic spectrum line into a few levels $\sigma_{H i}$ (Fig. 10). The third step is the calculation of the total number of stress cycles $n_{\Sigma}$ and dividing of this number into the cycles number of participating stress levels $n_{\Sigma i}$. In order to be comparable, Fig. 8 shows all load spectrums presented in the same scale of $10^{6}$ cycles and with a relative torque value of $x_{i}=T_{i} / T_{1 \max }=0 \sim 1$. For calculation purposes, Fig. 10 shows the relative scales transformed into the absolute. For a given gear pair for a vehicle service life of one million kilometres, the number of pinion revolutions $n_{\Sigma}$ is calculated and then divided into $n_{\Sigma I}$, i.e. $n_{\Sigma}=\Sigma n_{\Sigma i}$. The ratio between $\sigma_{H i}$ levels and $n_{\Sigma i}$ has to be maintained as shown in the load spectrums ratios of torques in Fig. 8.

\subsection{Decomposition of the Reliability System}

According to the reliability system presented in Fig. 6, the total reliability of the gearbox is $R=R_{g} \cdot R_{c} \cdot R_{b} \cdot R_{s e} \cdot R_{s t}$. The reliability of the gears contains the elementary reliability of all gear pairs against teeth flanks wear, i.e. $R_{g}=R_{g 0} \cdot R_{g 1} \cdot R_{g 2} \cdot R_{g 3}$. $\cdot R_{g 4} \cdot R_{g 5} \cdot R_{g R}$ and includes $j=7$ components. The reliability of couplings $R_{c}=R_{c 1} \cdot R_{c 2} \cdot R_{c 3}$ also contains $c=3$ components. Engagement of a gear set for reverse motion $\mathrm{R}$ is carried out without the coupling, using middle gear axial movement, carried out by the steering system. The reliability of the bearings is $R_{b}=R_{b 1} \cdot R_{b 2} \cdot R_{b 3} \cdot R_{b 4} \cdot R_{b 5}$, it contains $b=5$ elementary reliabilities, and the reliability of the seals $R_{s e}=R_{s e 1} \cdot R_{s e 2}$, se $=2$. The total number of elementary reliabilities, including the reliability of the steering assembly, is $r=g+c+b+s e+s t=7+3+5+2+1=18$.

The desired reliability, $R$, as a functional requirement after the desired service life has to be decomposed to the level of elementary reliabilities $R_{r}$ (Fig. 6). The simplest way to decompose it is in the form $R_{r}=R^{1 / r}$. The advantage of this calculation is the development of a design structure that is composed of components with the same level of reliabilities. The service life resources of all components will disappear at the same time. On the other hand, various combinations are possible.

\subsection{Load Capacity Calculation}

The calculation of load capacity implies that all design parameters, design properties of components, and characteristics of materials and parts are known and defined. The load capacity of the existing automotive gearbox is the result of load capacities for every speed in relation to the load spectrum. For a certain gear pair this relationship is:

$$
\begin{gathered}
\sigma_{H}=Z \sqrt{\frac{2 T_{1}}{b \cdot d_{1}^{2}} \frac{u+1}{u} K} \leq \sigma_{\text {Hdes }}, \\
T_{1}=\left(\sigma_{\text {Hdes }}^{2} \frac{d_{1}^{2}}{2 K Z^{2}} \frac{u}{u+1}\right) \cdot b=a \cdot b .
\end{gathered}
$$

In the above formulas $T_{1}$ is the torque at the pinion (lower gear in gear pair), $d_{1}$ is the pitch diameter of the pinion, $b$ is the gear pair width, $u=z_{2} / z_{1}$ is the transmission ratio, where $z_{1}$ is teeth number of the pinion, and $z_{2}$ teeth number of the gear. The load distribution at the gear teeth contacts and the load dynamics effect are included in value $K$, and the 
contact conditions are given by value $Z$, all according to ISO 6336 and DIN 3990 standards concerning helical and spur gear calculations. The value $a_{j j}$ in $\mathrm{Nm} / \mathrm{mm}$ denotes the member in the axiomatic matrix A and connects the functional requirement $T_{1 j}$ and the gear pair width design parameter $b_{j}$ (in $\mathrm{mm}$ ). The design allowable stress $\sigma_{\text {Hdesj }}$ is calculated in relation to the elementary reliability for a corresponding gear pair $j$ and all the material and operation characteristics including variation and randomness. For all gear pairs in the gearbox this relationship takes the form:

$$
\left\{\begin{array}{l}
T_{10} \\
T_{11} \\
T_{12} \\
T_{13} \\
T_{14} \\
T_{15} \\
T_{1 R}
\end{array}\right\}=\left[\begin{array}{ccccccc}
a_{00} & 0 & 0 & 0 & 0 & 0 & 0 \\
0 & a_{11} & 0 & 0 & 0 & 0 & 0 \\
0 & 0 & a_{22} & 0 & 0 & 0 & 0 \\
0 & 0 & 0 & a_{33} & 0 & 0 & 0 \\
0 & 0 & 0 & 0 & a_{44} & 0 & 0 \\
0 & 0 & 0 & 0 & 0 & a_{55} & 0 \\
0 & 0 & 0 & 0 & 0 & 0 & a_{R R}
\end{array}\right]\left\{\begin{array}{c}
b_{0} \\
b_{1} \\
b_{2} \\
b_{3} \\
b_{4} \\
b_{5} \\
b_{R}
\end{array}\right\} .
$$

These are torques at the pinions of the gear pairs. The next step is the recalculation of these torques at the input torque. When the load capacity of the gearbox is identified, the maximum input torque can be obtained. These data are suitable for analysis of the design parameters correlation and optimality under the chosen operation conditions.

\subsection{Load capacity and DP Harmonization}

The design parameters definition of gear transmission units for common application is presented in [5] and geometric parameterization of gears is processed. One of the points of this paper is successive calculation of the transformation matrix members in order to decouple them, i.e. decoupling the transformation matrix according to the axiomatic rule. In this way, the problem of complex interrelation between design parameters (DP) of gears, shafts and bearings is solved. Eq. (5) represents the relationship between the DP and FR of independent gear pairs that operate separately when engaged by a corresponding coupling, and this is the reason why transformation matrix $\mathbf{A}$ is simply decoupled. The calculation of other DPs such as coupling dimensions, shafts dimensions, bearing dimensions or load capacity is made possible by applying the successive calculation procedure presented in [5].

\section{Computer Program Description}

The design parameters definition (DPD) is the result of satisfying functional requirements (FR) according to the presented methodology. Furthermore, the design parameters have to be determined with regard to numerous additional limitations, such as volume minimization, suspension, vibration and noise level minimization and insulation, cost minimization, modularity provision, i.e. the automotive gearbox is compatible with the design module in various vehicles, etc. For this purpose, axiomatic and robust methodology should be combined with interactive decision-making and iterative repetition of these calculations in order to obtain maximal harmony of the DP with the desired functional requirements and design constraints. The computer program DRAG has been developed for that purpose.
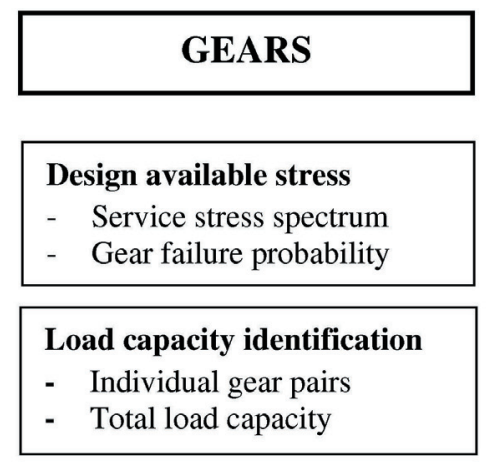

Design parameters definition

- Gear diameters (center dist.)

- Gear width
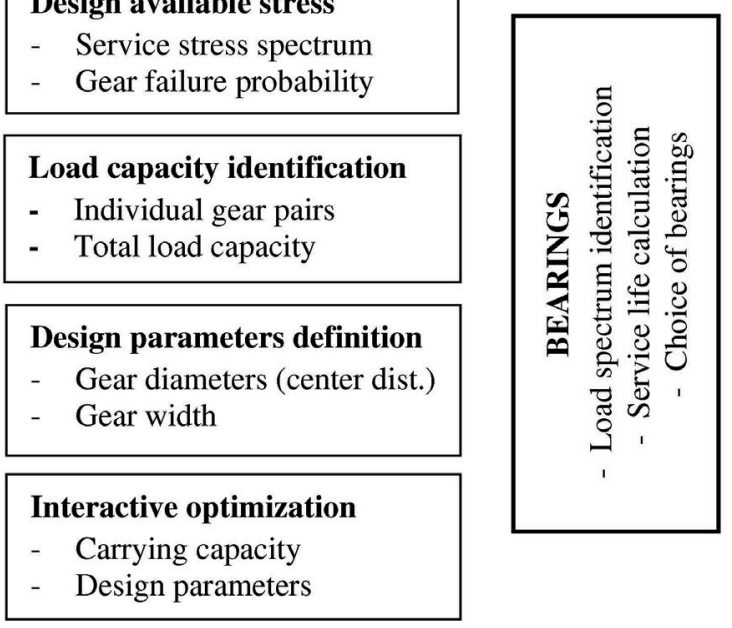

Fig. 11. Modules of DRAG software for automotive gearbox optimization

Fig. 11 shows the DRAG (Design for Reliability of Automotive Gearboxes) program structure. The program consists of five modules with tasks that require communication with a designer in order to be solved. The first of these is the module defining the gear flank allowable stress (design Hertzian stress $\left.\sigma_{\text {Hdes }}\right)$. This module contains a complex calculation of the elementary reliability of gears based on the operation load (operation stress) spectrum and possible failure probability (Fig. 10). The calculation iterates until the calculated elementary reliability 
becomes equal to the elementary reliability obtained by the deduction of the desired total gearbox reliability at the end of service life. The maximal stress level in the last iteration is $\sigma_{H d e s}$. The input data for these calculations are the operation load spectrum, the gear pair service life, and the range of gear flank failure probability distribution. The load spectrum is the result of various combinations of operation conditions of the automotive gearbox and service life. The failure probability range is the result of extensive laboratory testing or data presented in DIN 3990 or ISO 6336. An interactive variation of operation conditions and the desired reliability produces a variation of $\sigma_{H d e s}$, which provides robustness for the further calculated DP or FR.

The second module shown in Fig. 11 is the load capacity identification of the automotive gearbox. The central relationship in this module is the matrix (axiomatic) Eq. (5) which defines the individual load capacity of gear pairs in the automotive gearbox. The first stage in this process is the calculation of values of matrix members $a$, which correlated with the already identified design stress $\sigma_{\text {Hdes }}$, gear pair dimensions and other influences presented by Eq. (4). The calculated load capacities $T_{1 i}$ of individual gear pairs are not the load capacity of the complete gearbox. These represent the basis for calculating the total value of input $T_{\text {input }}$ and output torque $T_{\text {output }}$ for every level of gearbox speed. The minimum value of the input torque represents the load capacity of the gearbox. The optimal design of the gearbox with design parameters harmonised with the operation conditions has similar values of input torque for every speed level.

For the purpose of the existing gearbox redesign or new design development, it is necessary to define the new design parameters (DP) for the desired load capacity and for other functional requirements (FR). This is shown in the third software module in Fig. 11. The calculation process is reversed in the second module. The input torque (gearbox load capacity) is decomposed to the level of the individual gear pair and then follows the inverse calculation according to Eq. (5). This step has two possibilities. If the centre distance and diameters of the gear pairs are fixed, the results of the calculation are the gear widths of individual gear pairs. The second possibility is the calculation of the necessary gear diameters with a defined relationship between the gear diameter and gear width. Using the results of the gear diameters calculation, it is necessary to decide upon the same centre distance of all gear pairs and then repeat the calculation in order to change the gear pair widths if necessary. According to the presented procedure, this module is very interactive and needs numerous decisions and input data for further calculation.

\section{Experimental Data and Computer Program Verification}

The last module for gear calculation given in Fig. 11 is the module for interactive optimization. Calculations contained in the previous two modules, as a rule, give no harmonised design parameters or load capacities of individual gear pairs. It is possible to obtain the optimal design structure or full design utility if the load capacities and design parameters of the individual design components are harmonised between each other and with the operation conditions. The gearbox load capacity (input torque) calculated based on the load capacity of individual gear pairs, can be a variable value. Harmonization means calculation of the design parameters, e.g. the gear width, in order to obtain the equilibrium of input torques, which is calculated based on the load capacities of individual gear pairs. This is also a harmonization of design parameters with operation conditions, i.e. with the functional requirements that operation conditions produce. The case study is carried out using the existing FAMOS six-speed gearbox which is mounted in trucks FAP 1620, 1921, 2021, TAM-170 and in bus transmission FAP 537.3. Table 2 presents the calculated load capacities (input torques) for the design parameters of this gearbox and Figs. 7 and 8 show the operation conditions. The load capacities of individual gear pairs are also presented. There is a great variation in input torque $T_{\text {input }}$. The load capacity of individual gear pairs is not harmonised with the load spectrums for the operation conditions used in the calculation (middle conditions).

Table 2. Load capacity of individual gear pairs for DP not harmonized with operation conditions

\begin{tabular}{cccc}
\hline $\begin{array}{c}\text { Gear } \\
\text { pair }\end{array}$ & $\begin{array}{c}\text { Gear width } b \\
{[\mathrm{~mm}]}\end{array}$ & $\begin{array}{c}\text { Gear pair load } \\
\text { capacity } T_{1}[\mathrm{Nm}]\end{array}$ & $\begin{array}{c}\text { Gearbox load capacity } \\
T_{\text {input }}[\mathrm{Nm}]\end{array}$ \\
\hline 0 & 35 & 1000 & 1000 \\
\hline 1 & 44 & 1200 & 746 \\
\hline 2 & 32 & 1100 & 683 \\
\hline 3 & 31 & 1367 & 849 \\
\hline 4 & 27.5 & 950 & 659 \\
\hline 5 & 32.5 & 600 & 825 \\
\hline$R$ & 57 & 1250 & 776 \\
\hline
\end{tabular}

Table 3 shows the result, i.e. the new values of the gear widths of individual gear pairs, which is harmonized to the operation conditions for nominal input torques 750 and $850 \mathrm{Nm}$. For this purpose, the middle operation regime and the total reliability 
of gearbox 0.92 (for individual gear pair 0.99) after one million kilometres of service life were used. Gears are made from steel $15 \mathrm{CrNi} 6$ with carbonised flanks. A lower level of reliability and easier operation conditions can provide a higher level of gearbox load capacity. The DRAG software allows for the analysis of data varying trends at an intermediary point between modules. These variations are the result of the total operation conditions decomposition to the level of individual gearbox component operation conditions. This, however, requires a separate analysis of correlations between operation conditions and the design allowable stresses, FR and DP.

Table 3. Gearbox DP harmonized with operation conditions for load capacity 750 and $850 \mathrm{Nm}$

\begin{tabular}{ccccc}
\hline \multirow{2}{*}{$\begin{array}{c}\text { Gear } \\
\text { pair }\end{array}$} & $\begin{array}{c}\text { Gearbox load capacity for all } \\
\text { speeds } T_{\text {input }}=750 \mathrm{Nm}\end{array}$ & $\begin{array}{c}\text { Gearbox load capacity for all } \\
\text { speeds } T_{\text {input }}=850 \mathrm{Nm}\end{array}$ \\
\cline { 2 - 5 } & $\begin{array}{c}\text { Gear width } \\
b \text { [mm] }\end{array}$ & $\begin{array}{c}\text { Gear pair load } \\
\text { capacity } T_{1}[\mathrm{Nm}]\end{array}$ & $\begin{array}{c}\text { Gear width } \\
b[\mathrm{~mm}]\end{array}$ & $\begin{array}{c}\text { Gear pair load } \\
\text { capacity } T_{1}[\mathrm{Nm}]\end{array}$ \\
\hline 0 & 26 & 750 & 30 & 850 \\
\hline 1 & 44 & 1206 & 50 & 1367 \\
\hline 2 & 35 & 1206 & 39 & 1367 \\
\hline 3 & 27 & 1206 & 31 & 1367 \\
\hline 4 & 31 & 1081 & 35 & 1225 \\
\hline 5 & 29 & 545 & 33 & 618 \\
\hline $\mathrm{R}$ & 55 & 1206 & 62 & 1367 \\
\hline
\end{tabular}

The presented procedure provides a harmonisation of design parameters with operation conditions. As it is impossible to adapt the design parameters to individual customer needs, the procedure is available for reverse application. Design parameters fall in the middle of the range of their variation. Individual customers may need gearboxes for various operation conditions. The load capacity can be calculated for each of them. For difficult conditions the load capacity has a lower value and for easier conditions this value is higher. The software module for the bearings calculation (Fig. 11) is not directly coupled with modules for the calculation and design parameters harmonisation of gear pairs. The harmonisation of gear parameters, coupling and shafts parameters (dimensions) is followed by the identification of the individual load spectrum for each bearing according to operation conditions. These spectrums present the basis for the service life calculation of each of the five bearings or the opposite, for the individual bearing DP definition. The constraints for this calculation are the available space, elementary reliability, etc. Software interactivity allows users to make key decisions, such as choosing the combination of operating conditions, choosing the characteristics of components (material, thermal treatment, technology processing, etc.), as well as selection of component design parameters, automotive gearbox service life, and reliability level at the end of service life, etc. The DRAG software is a tool for automotive gearbox harmonisations with the desired operating conditions, i.e. the load capacity and design parameters by applying the robust design and property-based design methodology.

\section{CONCLUSIONS}

The main objective of this paper is to develop a procedure that can correlate the functional requirements, random operation conditions and other limitations accordingly to the design parameters and provide a model for the design of a certain mechanical structure. The following contributions have been presented:

- Integration of robust and axiomatic design methodology and design-based properties.

- Reliability is used as the design requirement, the design components property, and as the quality and design behaviour indicator. therefore, a specific approach for defining elementary reliability is established. This reliability is a complex probability made up of operation conditions (load) probability and the failure probability of components exposed to a certain load level.

- As a case study, the automotive gearbox is decomposed into components and the system of reliability is structured to the level of elementary reliability, which represents a design property of automotive components and a design constraint.

- Random operation conditions of the gearbox are included by using load spectrums, which are designed incorporating the results of the torque measurement, the users' interview method and the assessment. Load spectrums and gearbox speed participation in the course of service life provide robustness to the approach.

- The computer program for load capacity and design parameter calculation of automotive gearboxes for random operation conditions is developed. The DRAG program is based on the developed model, which provides robustness to the results.

\section{ACKNOWLEDGMENT}

This article is a contribution to the Ministry of Education and Science of Serbia funded project TR 035006 . 


\section{REFERENCES}

[1] Suh, N. (2007). Ergonomics, axiomatic design and complexity theory. Theoretical Issues in Ergonomics Science, vol. 8, no. 2, p. 101-121, DOI:10.1080/14639220601092509.

[2] Hu, Y., Rao, S. (2011). Robust Design of Horizontal Axis Wind Turbines Using Taguchi Method. Journal of Mechanical Design, vol. 133, no. 11, paper: 111009.

[3] Saha, A., Ray, T. (2011). Practical Robust Design Optimization Using Evolutionary Algorithms. Journal of Mechanical Design, vol. 133, no. 10, paper 101012.

[4] Du, X. (2012). Toward Time-Dependent Robustness Metrics. Journal of Mechanical Design, vol. 134, no. 1, paper: 011004.

[5] Ognjanovic, M., Benur, M. (2011). Experimental research for robust design of power transmission components. Meccanica, vol. 46, p. 699-710, DOI:10.1007/s11012-010-9331-y.

[6] Dersjo, T., Olsson, M. (2011). Reliability based design optimization using a single constraint approximation poin. Journal of Mechanical Design, vol. 133, no. 3, paper: 031006.

[7] Moura, M., Zio, E., Lins, I., Droguett, E. (2011). Failure and reliability prediction by support vector machines regression of time series data. Reliability Engineering and System Safety, vol. 96, p.1527-1534, DOI:10.1016/j.ress.2011.06.006.

[8] Lisnianski, A., Elmakias, D., Laredo, D., Haim, H.B. (2012). A multi-state Markov model for a shortterm reliability analysis of a power generating unit. Reliability Engineering and System Safety, vol. 98, p.16, DOI:10.1016/j.ress.2011.10.008.

[9] Natvig, B., Huseby, A., Reistadbakk, M. (2011). Measures of component importance in repairable multistate systems - a numericalstudy. Reliability Engineering and System Safety, vol. 96, p. 1680-1690, DOI:10.1016/j.ress.2011.07.006.

[10] Noh, Y., Choi, K., Lee, I. (2011). Reliability-based design optimization with confidence level for nonGaussian distributions using bootstrap method. Journal of Mechanical Design, vol. 133, no. 9, paper: 091001.

[11] Jiang, R., Murthy, P. (2011). A study of Weibull shape parameter: Properties and significance. Reliability Engineering and System Safety, vol. 96, p. 1619-1626, DOI:10.1016/j.ress.2011.09.003.

[12] Murthy, P., Rausand, M., Virtanen, S. (2009). Investment in new product reliability. Reliability Engineering and System Safety, vol. 94, no. 10, p. 1593-1600, DOI:10.1016/j.ress.2009.02.031.

[13] Li, S. (2011). A matrix-based clustering approach for the decomposition of design problems. Research in Engineering Design, vol. 22, p. 263-278, DOI:10.1007/ s00163-011-0111-z.

[14] Minguez, R., Conejo, A., Garcia-Bertrand, R. (2011). Reliability and decomposition techniques to solve certain class of stochastic programming problems.
Reliability Engineering and System Safety, vol. 96, p. 314-323, DOI:10.1016/j.ress.2010.09.011.

[15] Wang, Y., He, Z., Xiang, J., Zi, Y. (2012). Application of local mean decomposition to the surveillance and diagnostics of low-speed helical gearbox. Mechanism and Machine Theory, vol. 47m p. 62-73, DOI:10.1016/j. mechmachtheory.2011.08.007.

[16] Osman, K., Stamenkovic, D., Lazarevic, M. (2011). Robust product architecture development combining matrix-based approaches and function-based failure propagation method - M-FBFP Framework. FME Transactions, vol. 39, no. 145-156.

[17] Holt, R., Barnes, C. (2010). Towards an integrated approach to "Design for X": an agenda for decisionbased DFX research. Research in Engineering Design, vol. 21, p. 123-136, DOI:10.1007/s00163-009-0081-6.

[18] Krehmer, H., Meerkamm, H., Wartzack, S. (2011). Monitoring a property based product development from requirements to a mature product, e-Proceedings of the International Conference on Engineering Design, Copenhagen.

[19] Raffaeli, R., Mengoni, M., Germani, M. (2010). A software system for "Design for X" impact evaluations in redesign processes, Strojniški vestnik - Journal of Mechanical Engineering, vol. 56, no. 11, p. 707-717.

[20] Milutinović, M., Ognjanović, M. (2009). Failure probability of gear drives components and reliable fatigue life estimation. Proceedings of the 3 rd International Conference on Power Transmission, Kallithea, p. 285-290.

[21] Ognjanovic, M., Milutinovic, M. (2010). Careing capacity model of automative gearboxes based on reliabilty as design constraint. Proceedings of the International Conference on Gears, Munich, p. 13771380.

[22] Curtis, S., Harston, S., Mattson, C. (2011). The fundamentals of barriers to reverse engineering and their implementation into mechanical components. Research in Engineering Design, vol. 22, p. 245-261, DOI:10.1007/s00163-011-0109-6.

[23] Nikolic, V., Dolicanin, C., Dimitrijevic, D. (2012). Dynamic model for the stress and strain state analysis of a spur gear transmission. Strojniški vestnik - Journal of Mechanical Engineering, vol. 58, no. 1, p. 56-67.

[24] Shim, Y., Kauh, S.K., Ha, K. (2011). Evaluation of idle stability through in-situ torque measurement in automatic transmission vehicles. International Journal of Automotive Technology, vol. 12, no. 3, p. 315-320, DOI:10.1007/s12239-011-0037-5.

[25] Irimescu, A., Mihon, L., Pãdure, G. (2011). Automotive transmission efficiency measurement using a chassis dynamometer. International Journal of Automotive Technology, vol. 12, no. 4, p. 555-559, DOI:10.1007/ s12239-011-0065-1.

[26] Hong, M., Ouyang, M., Shen, T. (2011). Torque-based optimal vehicle speed control. International Journal of Automotive Technology, vol. 12, no. 1, p. 45-49, DOI:10.1007/s12239-011-0006-z. 